The Optimal Projection Equations for Reduced-Order, Discrete-Time Modeling, Estimation, and Control D.S. Bernstein, L.D. Davis, D.C. Hyland 


\title{
The Optimal Projection Equations for Reduced-Order, Discrete-Time Modeling, Estimation, and Control
}

\author{
Dennis S. Bernstein, ${ }^{*}$ Lawrence D. Davis, $\dagger$ and David C. Hyland $\ddagger$ \\ Harris Corporation, Melbourne, Florida
}

\begin{abstract}
The optimal projection equations derived previously for reduced-order, continuous-time modeling, estimation, and control are developed for the discrete-time case. The design equations are presented in a concise, unified manner to facilitate their accessibility for the development of numerical algorithms for practical applications. As in the continuous-time case, the standard Kalman filter and linear-quadratic-Gaussian results are immediately obtained as special cases of the estimation and control results.
\end{abstract}

\section{Nomenclature}

$$
\begin{aligned}
& A, B, C=n \times n, n \times m, \ell \times n \text { matrices } \\
& A_{m}, B_{m}, C_{m}=n_{m} \times n_{m}, n_{m} \times m, \ell \times n_{m} \text { matrices } \\
& A_{e}, B_{e}, C_{e}, D_{e}=n_{e} \times n_{e}, n_{e} \times \ell, p \times n_{e}, p \times \ell \text { matrices } \\
& A_{c}, B_{c}, C_{c}, D_{c}=n_{c} \times n_{c}, n_{c} \times \ell, m \times n_{c}, m \times \ell \text { matrices } \\
& E_{i} \quad=\text { matrix with unity in the }(i, i) \text { position } \\
& \boldsymbol{E} \quad=\text { expected value } \\
& I_{r} \quad=r \times r \text { identity matrix } \\
& k \quad=\text { discrete-time index } 1,2,3, \ldots \\
& L \quad=p \times n \text { matrix } \\
& n, m, \ell, n_{m} \text {, } \\
& n_{e}, n_{c}, p=\text { positive integers, } 1 \leq n_{m}, n_{e}, n_{c} \leq n \\
& R, \stackrel{N}{N}, R_{2}=\ell \times \ell, p \times p, m \times m \text { positive-definite matrices } \\
& R_{1} \quad=n \times n \text { nonnegative-definite matrix } \\
& R_{12} \quad=n \times m \text { matrix such that } R_{1}-R_{12} R_{2}^{-1} R_{12}^{T} \\
& \text { is nonnegative definite } \\
& \boldsymbol{R}, \boldsymbol{R}^{r \times s} \quad=\text { real numbers, } r \times s \text { real matrices } \\
& \tilde{R} \quad=\left[\begin{array}{cc}
R_{1} & R_{12} \\
R_{12}^{T} & R_{2}
\end{array}\right] \\
& \operatorname{tr} Z \quad=\text { trace of square matrix } Z \\
& u, y, y_{e} \quad=m-, \ell-, p \text {-dimensional vectors } \\
& V \quad=m \times m \text { positive-definite covariance of } w \\
& V_{1} \quad=n \times n \text { nonnegative-definite covariance of } w_{1} \\
& V_{2} \quad=\ell \times \ell \text { positive-definite covariance of } w_{2} \\
& V_{12} \quad=n \times \ell \text { cross-covariance of } w_{1}, w_{2} \\
& w, w_{1}, w_{2}=m-, n-, \ell \text {-dimensional zero-mean discrete-time } \\
& \text { white noise processes } \\
& x, x_{m}, x_{e}, x_{c}=n-, n_{m^{-}}, n_{e^{-}}, n_{c} \text {-dimensional vectors } \\
& Z_{(i, j)} \quad=(i, j) \text { element of matrix } Z \\
& Z^{T} \quad=\text { transpose of vector or matrix } Z \\
& Z^{-T} \quad=\left(Z^{T}\right)^{-1} \text { or }\left(Z^{-1}\right)^{T} \\
& \Pi_{i}(\Psi) \quad=\Psi E_{i} \Psi^{-1} \text { (unit-rank eigenprojection }{ }^{47} \text { ) } \\
& \rho(Z) \\
& =\text { rank of matrix } Z
\end{aligned}
$$

Received April 1, 1985; revision received Aug. 26, 1985. Copyright (c) American Institute of Aeronautics and Astronautics, Inc., 1986. All rights reserved.

*Associate Principal Engineer, Controls Analysis and Synthesis Group. Government Aerospace Systems Division.

†Lead Engineer, Controls Analysis and Synthesis Group, Government Aerospace Systems Division. Member AIAA.

$\ddagger$ Principal Engineer, Controls Analysis and Synthesis Group. Government Aerospace Systems Division. Member AIAA.

\section{Introduction}

$\mathrm{N}$ a recent series of papers ${ }^{1-11}$ it has been shown that the first-order necessary conditions for quadratically optimal, continuous-time, reduced-order modeling, estimation, and control can be transformed into coupled systems of two, three, and four matrix equations, respectively. This coupling, due to the presence of an oblique projection (idempotent matrix), arises as a rigorous consequence of optimality, hence suggesting the name optimal projection. For the estimation and control problems, this formulation provides a direct generalization of classical steady-state Kalman filter and linearquadratic-Gaussian (LQG) control theory. In the full-order case the projection becomes the identity matrix, the additional two modified Lyapunov equations drop out, and the remaining modified Riccati equations become the usual Riccati equations.

Coupling via the optimal projection supports the view that sequential reduced-order design procedures consisting of either 1) model reduction followed by estimator (controller) design or 2) estimator (controller) design followed by estimator (controller) reduction are generally not optimal. Furthermore, for the control problem the coupled structure of the equations yields the further insight that in the reduced-order case there is no longer separation between the operations of state estimation and state-estimate feedback, i.e., the certainty equivalence principle breaks down.

For practical applications, the optimal projection equations permit the development of alternative numerical algorithms that operate through successive iteration of the optimal projection ${ }^{4.6}$ rather than by gradient search techniques. ${ }^{12}$ By recognizing that each local extremalcorresponds to $n_{r}$ possible choices out of $n$ rank-1 eigenprojections of the product of a pair of pseudogramians, it is possible to efficiently identify the global minimum. ${ }^{10}$ This idea is philosophically similar to Skelton's component-cost analysis. ${ }^{13-15}$

The purpose of the present paper is to develop the optimal projection equations for reduced-order modeling, estimation, and control in the discrete-time case. Since the underlying theory has been discussed previously, ${ }^{1-11}$ the presentation herein is geared toward a clear and concise statement of the main results to facilitate numerical developments and practical application. For example, by expressing the optimal projection in terms of eigenprojections, a variety of novel algorithms are immediately suggested. For illustrative purposes we apply the results on reduced-order state estimation to a third-order problean to obtain reduced-order estimators and the results on reduced-order dynamic compensation to a tenth-order problem to obtain reduced-order controllers. 
Because of the discrete-time setting it is now possible to permit static feedthrough gains in the estimator and controller designs. As previously noted, ${ }^{7}$ nonsingular control weighting and measurement noise in the continuous-time case permit only a purely dynamic (strictly proper) controller. Note that this is precisely the case in continuous-time LQG theory, which always yields a strictly proper feedback controller. The static gains in the discrete-time estimator problem permit simultaneous, unified treatment of nondynamic least-squares estimation along with dynamic (Kalman filter-type) estimation.

The references include a representative sampling of papers on quadratically optimal reduced-order modeling, ${ }^{13-25}$ estimation, ${ }^{25-33}$ and control, ${ }^{34-46}$ along with closely related approaches. For emphasis on the discrete-time problem, see Refs. 18, 30, 41, 42, 44, and 45 .

\section{Problem Statement and Main Results}

We now state the reduced-order modeling, estimation, and control problems. The object of the model-reduction problem is to determine a model of reduced state-space dimension whose steady-state response to white noise inputs (or, equivalently, impulse response) best approximates, in a quadratic (least-squares) sense, the response of a given high-order system. In the reduction process the order of the reduced model is fixed and the optimization is performed over the model parameters.

\section{Reduced-Order Modeling Problem}

Given the model

$$
\begin{gathered}
x(k+1)=A x(k)+B w(k) \\
y(k)=C x(k)
\end{gathered}
$$

design a reduced-order model

$$
\begin{gathered}
x_{m}(k+1)=A_{m} x_{m}(k)+B_{m} w(k) \\
y_{m}(k)=C_{m} x(k)
\end{gathered}
$$

which minimizes the model-reduction criterion

$$
\begin{aligned}
& J_{m}\left(A_{m}, B_{m}, C_{m}\right) \\
& \quad \triangleq \lim _{k \rightarrow \infty} \boldsymbol{E}\left[y_{m}(k)-y(k)\right]^{T} R\left[y_{m}(k)-y(k)\right]
\end{aligned}
$$

The goal of the reduced-order state-estimation problem is to design an estimator of given order which yields quadratically optimal (least squares) estimates of specified linear combinations $L x$ of states $x$. In practice, the order of the estimator may be determined by implementation constraints, such as real-time computing capability. Note that the feedthrough term $D_{e}$ permits the utilization of a static least-squares estimator in conjunction with the dynamic estimator $\left(A_{e}, B_{e}, C_{e}\right)$.

\section{Reduced-Order State-Estimation Problem}

Given the observed system

$$
\begin{gathered}
x(k+1)=A x(k)+w_{1}(k) \\
y(k)=C x(k)+w_{2}(k)
\end{gathered}
$$

design a reduced-order state estimator

$$
\begin{gathered}
x_{e}(k+1)=A_{e} x_{e}(k)+B_{e} y(k) \\
y_{e}(k)=C_{e} x_{e}(k)+D_{e} y(k)
\end{gathered}
$$

which minimizes the state-estimation criterion

$$
\begin{aligned}
& J_{e}\left(A_{e}, B_{e}, C_{e}, D_{e}\right) \\
& \quad \triangleq \lim _{k \rightarrow \infty} \boldsymbol{E}\left[y_{e}(k)-L x(k)\right]^{T} N\left[y_{e}(k)-L x(k)\right]
\end{aligned}
$$

For the fixed-order dynamic-compensation problem, a static feedthrough term is included, i.e., the controller may be nonstrictly proper.

\section{Reduced-Order Dynamic-Compensation Problem}

Given the controlled system

$$
\begin{gathered}
x(k+1)=A x(k)+B u(k)+w_{1}(k) \\
y(k)=C x(k)+w_{2}(k)
\end{gathered}
$$

design a reduced-order dynamic compensator

$$
\begin{gathered}
x_{c}(k+1)=A_{c} x_{c}(k)+B_{c} y(k) \\
u(k)=C_{c} x_{c}(k)+D_{c} y(k)
\end{gathered}
$$

which minimizes the dynamic-compensation criterion

$$
\begin{gathered}
J_{c}\left(A_{c}, B_{c}, C_{c}, D_{c}\right) \triangleq \lim _{k \rightarrow \infty} E\left[x(k)^{T} R_{1} x(k)\right. \\
\left.+2 x(k)^{T} R_{12} u(k)+u(k)^{T} R_{2} u(k)\right]
\end{gathered}
$$

To guarantee that $J_{m}, J_{e}$, and $J_{c}$ are finite and independent of initial conditions, consideration is restricted to the following (open) sets. [A triple $(\hat{A}, \hat{B}, \hat{C})$ is minimal if $(\hat{A}, \hat{B})$ is controllable and $(\hat{A}, \hat{C})$ is observable.]

$$
\begin{aligned}
& \mathscr{S}_{m} \triangleq\left\{\left(A_{m}, B_{m}, C_{m}\right):\right. \\
& \left.A_{m} \text { is stable and }\left(A_{m}, B_{m}, C_{m}\right) \text { is minimal }\right\} \\
& \mathscr{S}_{e} \triangleq\left\{\left(A_{e}, B_{e}, C_{e}, D_{e}\right):\right. \\
& \left.A_{e} \text { is stable and }\left(A_{e}, B_{e}, C_{e}\right) \text { is minimal }\right\} \\
& \mathscr{S}_{c} \triangleq\left\{\left(A_{c}, B_{c}, C_{c}, D_{c}\right):\right. \\
& \left.\left[\begin{array}{cc}
A+B D_{c} C & B C_{c} \\
B_{c} C & A_{c}
\end{array}\right] \text { is stable and }\left(A_{c}, B_{c}, C_{c}\right) \text { is minimal }\right\}
\end{aligned}
$$

Let $n_{r}$ generically denote $n_{m}, n_{e}$, and $n_{c}$. The following factorization lemma will be needed for the main results.

Lemma 2.1. Let $\tau \in \boldsymbol{R}^{n \times n}$. Then

$$
\begin{gathered}
\tau^{2}=\tau \\
\rho(\tau)=n_{r}
\end{gathered}
$$

if, and only if, there exist $G, \Gamma \in \boldsymbol{R}^{n_{r} \times n}$ such that

$$
\begin{gathered}
G^{T} \Gamma=\tau \\
\Gamma G^{T}=I_{n_{r}}
\end{gathered}
$$

Furthermore, $G$ and $\Gamma$ are unique to a change of basis in $\boldsymbol{R}^{n_{r}}$.

Proof. Sufficiency is obvious. To prove necessity, first note that due to Eq. (16) the eigenvalues of $\tau$ are either 0 or 1 . Further, it is easy to see that $\tau$ has a diagonal Jordan canonical form. Hence, the result follows from

$$
\tau=S\left[\begin{array}{cc}
I_{n_{r}} & 0 \\
0 & 0
\end{array}\right] S^{-1}=G^{T} \Gamma
$$

where $G=\left[\begin{array}{ll}\phi^{T} & 0\end{array}\right] S^{T}, \Gamma=\left[\begin{array}{ll}\phi^{-1} & 0\end{array}\right] S^{-1}$, and $\phi \in \boldsymbol{R}^{n_{r} \times n_{r}}$.

For convenience, call $G$ and $\Gamma$ satisfying Eqs. (18) and (19) a projective factorization of $\tau$. Furthermore, for $n \times n$ nonnegative-definite matrices (i.e., symmetric matrices with nonnegative eigenvalues) $\mathscr{Q}$ and $\mathscr{P}$, define the set of contragredi- 
ently diagonalizing transformations

$$
\begin{aligned}
& \mathscr{D}(\mathscr{Q}, \mathscr{P}) \\
& \quad \triangleq\left\{\Psi \in R^{n \times n}: \Psi^{-1} \mathscr{Q} \Psi^{-T} \text { and } \Psi^{T} \mathscr{P} \Psi \text { are diagonal }\right\}
\end{aligned}
$$

It follows from Ref. 48 , p. 123 , Theorem 6.2 .5 , that $\mathscr{D}(\mathscr{Q}, \mathscr{P})$ is always nonempty. This set does not, however, have a unique element since basis rearrangements and sign transpositions may be incorporated into $\Psi$. Further nonuniqueness arises if $2 \mathscr{P}$ has repeated eigenvalues.

Theorem 2.1. Suppose $A$ is stable and $\left(A_{m}, B_{m}, C_{m}\right) \in \mathscr{S}_{m}$ solves the reduced-order modeling problem. Then there exist $n \times n$ nonnegative-definite matrices $\hat{Q}$ and $\hat{P}$ such that $A_{m}$, $B_{m}$, and $C_{m}$ are given by

$$
\begin{aligned}
A_{m} & =\Gamma A G^{T} \\
B_{m} & =\Gamma B \\
C_{m} & =C G^{T}
\end{aligned}
$$

and such that $\hat{Q}$ and $\hat{P}$ satisfy

$$
\begin{aligned}
& \hat{Q}=A \tau \hat{Q} \tau^{T} A^{T}+B V B^{T} \\
& \hat{P}=A^{T} \tau^{T} \hat{P} \tau A+C^{T} R C
\end{aligned}
$$

where

$$
\tau \triangleq \sum_{i=1}^{n_{m}} \Pi_{i}(\Psi)
$$

for some $\Psi \in \mathscr{D}(\hat{Q}, \hat{P})$ such that $\left(\Psi^{-1} \hat{Q} \hat{P} \Psi\right)_{(i, i)} \neq 0, i=$ $1, \ldots, n_{m}$, and some projective factorization $G, \Gamma$ of $\tau$. Furthermore, the minimal cost is given by

$$
J_{m}\left(A_{m}, B_{m}, C_{m}\right)=\operatorname{tr}\left[\left(W_{c}-\tau \hat{Q} \tau^{T}\right) C^{T} R C\right]
$$

where $W_{c}$ is the unique (nonnegative-definite) solution of

$$
W_{c}=A W_{c} A^{T}+B V B^{T}
$$

For convenience in stating the estimator result, define the notation

$$
\begin{aligned}
& \Sigma_{Q} \triangleq\left(A Q C^{T}+V_{12}\right) \tilde{V}_{2}^{-1}\left(A Q C^{T}+V_{12}\right)^{T} \\
& \Sigma_{P} \triangleq\left(L-D_{e} C\right)^{T} N\left(L-D_{e} C\right) \\
& A_{Q} \triangleq A-\left(A Q C^{T}+V_{12}\right) \tilde{V}_{2}^{-1} C \\
& \tilde{V}_{2} \triangleq V_{2}+C Q C^{T} \\
& \tau_{\perp} \triangleq I_{n}-\tau
\end{aligned}
$$

Theorem 2.2. Suppose $A$ is stable and $\left(A_{e}, B_{e}, C_{e}, D_{e}\right) \in \mathscr{S}_{e}$ solves the reduced-order state-estimation problem. Then there exist $n \times n$ nonnegative-definite matrices $Q, \hat{Q}$, and $\hat{P}$ such that $A_{e}, B_{e}, C_{e}$, and $D_{e}$ are given by

$$
\begin{aligned}
& A_{e}=\Gamma\left[A-\left(A Q C^{T}+V_{12}\right) \tilde{V}_{2}^{-1} C\right] G^{T} \\
& B_{e}=\Gamma\left(A Q C^{T}+V_{12}\right) \tilde{V}_{2}^{-1} \\
& C_{e}=\left(L-D_{e} C\right) G^{T} \\
& D_{e}=L Q C^{T} \tilde{V}_{2}^{-1}
\end{aligned}
$$

and such that $Q, \hat{Q}$, and $\hat{P}$ satisfy

$$
\begin{aligned}
Q & =A Q A^{T}-\left(A Q C^{T}+V_{12}\right) \tilde{V}_{2}^{-1}\left(A Q C^{T}+V_{12}\right)^{T} \\
& +V_{1}+\tau_{\perp} \hat{Q} \tau_{\perp}^{T} \\
\hat{Q} & =A \tau \hat{Q} \tau^{T} A^{T}+\Sigma_{Q} \\
\hat{P} & =A_{Q}^{T} \tau^{T} \hat{P} A_{Q}+\Sigma_{P}
\end{aligned}
$$

where

$$
\tau \triangleq \sum_{i=1}^{n_{e}} \Pi_{i}(\Psi)
$$

for some $\Psi \in \mathscr{D}(\hat{Q}, \hat{P})$ such that $\left(\Psi^{-1} \hat{Q} \hat{P} \psi\right)_{(i, i)} \neq 0, \quad i=$ $1, \ldots, n_{e}$, and some projective factorization $G, \Gamma$ of $\tau$. Furthermore, the minimal cost is given by

$$
J_{e}\left(A_{e}, B_{e}, C_{e}, D_{e}\right)=\operatorname{tr}\left[\left(L Q L^{T}-D_{e} \tilde{V}_{2} D_{e}^{T}\right) N\right]
$$

For the control result, define the additional notation

$$
\begin{aligned}
& \Sigma_{Q}^{\prime} \triangleq\left(A Q C^{T}+V_{12}+B D_{c} \tilde{V}_{2}\right) \tilde{V}_{2}^{-1}\left(A Q C^{T}+V_{12}+B D_{c} \tilde{V}_{2}\right)^{T} \\
& \Sigma_{P}^{\prime} \triangleq\left(B^{T} P A+R_{12}^{T}+\tilde{R}_{2} D_{c} C\right)^{T} \tilde{R}_{2}^{-1}\left(B^{T} P A+R_{12}^{T}+\tilde{R}_{2} D_{c} C\right) \\
& A_{P} \triangleq A-B \tilde{R}_{2}^{-1}\left(B^{T} P A+R_{12}^{T}\right) \\
& \tilde{R}_{2} \triangleq R_{2}+B^{T} P B \\
& M \triangleq\left[\begin{array}{c}
I_{n} \\
D_{c} C
\end{array}\right], \quad \hat{M} \triangleq\left[\begin{array}{c}
I_{n} \\
-\tilde{R}_{2}^{-1}\left(B^{T} P A+R_{12}^{T}\right)
\end{array}\right]
\end{aligned}
$$

Theorem 2.3. Suppose $\left(A_{c}, B_{c}, C_{c}, D_{c}\right) \in \mathscr{S}_{c}$ solves the reduced-order dynamic-compensation problem. Then there exist $n \times n$ nonnegative-definite matrices $Q, P, \hat{Q}$, and $\hat{P}$ such that $A_{c}, B_{c}, C_{c}$, and $D_{c}$ are given by

$$
\begin{aligned}
A_{c}= & \Gamma\left[A-\left(A Q C^{T}+V_{12}\right) \tilde{V}_{2}^{-1} C\right. \\
& \left.-B \tilde{R}_{2}^{-1}\left(B^{T} P A+R_{12}^{T}\right)-B D_{c} C\right] G^{T} \\
B_{c}= & \Gamma\left[\left(A Q C^{T}+V_{12}\right) \tilde{V}_{2}^{-1}+B D_{c}\right] \\
C_{c}= & -\left[\tilde{R}_{2}^{-1}\left(B^{T} P A+R_{12}^{T}\right)+D_{c} C\right] G^{T} \\
D_{c}= & -\tilde{R}_{2}^{-1}\left(B^{T} P A Q C^{T}+R_{12}^{T} Q C^{T}+B^{T} P V_{12}\right) \tilde{V}_{2}^{-1}
\end{aligned}
$$

and such that $Q, P, \hat{Q}$, and $\hat{P}$ satisfy

$$
\begin{aligned}
Q= & A Q A^{T}-\left(A Q C^{T}+V_{12}\right) \tilde{V}_{2}^{-1}\left(A Q C^{T}+V_{12}\right)^{T} \\
& +V_{1}+\tau_{\perp} \hat{Q} \tau_{\perp}^{T} \\
P= & A^{T} P A-\left(B^{T} P A+R_{12}^{T}\right)^{T} \tilde{R}_{2}^{-1}\left(\dot{B}^{T} P A+R_{12}^{T}\right) \\
& +R_{1}+\tau_{\perp}^{T} \hat{P} \tau_{\perp} \\
\hat{Q}= & A_{P} \tau \hat{Q} \tau^{T} A_{P}^{T}+\Sigma_{Q}^{\prime} \\
\hat{P}= & A_{Q}^{T} \tau^{T} \hat{P} \tau A_{Q}+\Sigma_{P}^{\prime}
\end{aligned}
$$

where

$$
\tau \triangleq \sum_{i=1}^{n_{c}} \Pi_{i}(\Psi)
$$

for some $\Psi \in \mathscr{D}(\hat{Q}, \hat{P})$ such that $\left(\Psi^{-1} \hat{Q} \hat{P} \Psi\right)_{(i, i)} \neq 0, \quad i=$ $1, \ldots, n_{c}$, and some projective factorization $G, \Gamma$ of $\tau$. Further- 
more, the minimal cost is given by

$$
J_{c}\left(A_{c}, B_{c}, C_{c}, D_{c}\right)=\operatorname{tr}\left[\left(M Q M^{T}+\hat{M} \tau \hat{Q} \tau^{T} \hat{M}^{T}\right) \tilde{R}\right]
$$

Remark 2.1. To specialize the estimation and control results to the strictly proper (no-feedthrough) case, merely ignore Eqs. (30) and (39) and set $D_{e}=0$ and $D_{c}=0$ wherever they appear.

Remark 2.2. In the full-order cases $n_{e}=n$ and $n_{c}=n$ in Theorems 2.2 and 2.3 , the projection $\tau$ becomes the identity and Eqs. (32), (33), (42), and (43) play no role. In this case $G$ and $\Gamma$ are also the identity. Specializing further to the purely dynamic case $D_{e}=0, D_{c}=0$ as in the previous remark yields the standard Kalman filter and LQG results.

Remark 2.3. As previously noted, ${ }^{7,10,11}$ the indeterminacy in specifying the projective factorization $G, \Gamma$ satisfying Eqs. (18) and (19) corresponds to nothing more than an arbitrary choice of internal state-space basis for the design systems $\left(A_{m}, B_{m}, C_{m}\right),\left(A_{e}, B_{e z} C_{e}\right)$, and $\left(A_{c}, B_{c}, C_{c}\right)$.

Remark 2.4. Since $Q$ and $\hat{P}$ are balanced by means of the transformation $\Psi \in \mathscr{D}(\hat{Q}, \hat{P})$, it follows that $\Psi^{-1} \hat{Q} \hat{P} \Psi$ is diagonal. Hence, $\hat{Q} \hat{P}$ is semisimple and thus $\Pi_{i}(\Psi)$ is a rank-1 eigenprojection of $\hat{Q} \hat{P}$. (A semisimple matrix possesses a diagonal Jordan form. ${ }^{47,48}$ ) Although the optimal projection $\tau$ is characterized in Eqs. (25), (34), and (44) as the sum of rank-1 eigenprojections of $\hat{Q} \hat{P}$, because of the nonuniqueness in $\mathscr{D}(\hat{Q}, \hat{P})$, the theorems do not specify which eigenprojections actually comprise $\tau$. From analytical examples ${ }^{10}$ it can be seen that each of the $\left(\begin{array}{l}n \\ n_{r}\end{array}\right)$ possible projections may correspond to a local extremal in the optimization problem.

Remark 2.5. The proofs of Theorems 2.1-2.3 are similar to the continuous-time results and, hence, have been omitted. To help the reader reconstruct the lengthy manipulations, the key details differing from the continuous-time case are pointed out. For the control problem, an $\left(n+n_{c}\right) \times\left(n+n_{c}\right)$ discretetime algebraic Lyapunov equation is obtained for the steadystate covariance of the closed-loop system. Regarding this equation as a side constraint, the Lagrange multiplier technique is used to compute stationarity conditions that yield explicit expressions for $A_{c}, B_{c}, C_{c}$, and $D_{c}$. The projection arises when these expressions are substituted into the original

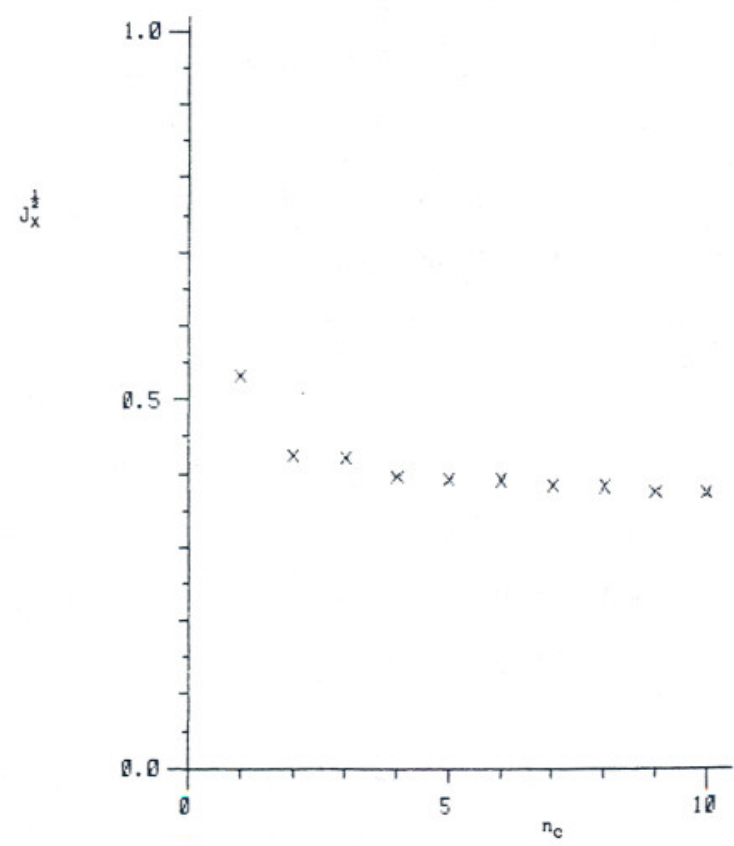

Fig. 1 Root-mean-square performance vs controller order for five-mode beam example. augmented Lyapunov equation and its dual. The interesting aspect is that the explicit gain expressions and the definition of the optimal projection arise in the reverse order as compared to the continuous-time derivation. Similar remarks apply to the reduced-order modeling and estimation problems.

\section{Examples}

As an application of Theorem 2.2 on reduced-order state estimation, the stirred-tank example from Ref. 36, pp. 107, 473 , and 531 , is considered. Ignoring the undisturbed volume state, the remaining states are the incremental tank concentration and variations in the feed concentrations. The problem data are as follows:

$$
\begin{aligned}
A & =\left[\begin{array}{lll}
0.9048 & 0.06702 & 0.02262 \\
0 & 0.8825 & 0 \\
0 & 0 & 0.9048
\end{array}\right] \\
C & =\left[\begin{array}{lll}
1 & 0 & 0
\end{array}\right], V_{2}=10^{-6}, V_{12}=0, N=L=I_{3} \\
V_{1} & =\left[\begin{array}{lll}
5.399 \times 10^{-6} & 8.015 \times 10^{-5} & 8.762 \times 10^{-5} \\
8.015 \times 10^{-5} & 2.212 \times 10^{-3} & 0 \\
8.762 \times 10^{-5} & 0 & 7.251 \times 10^{-3}
\end{array}\right]
\end{aligned}
$$

The standard Kalman filter result is

$$
\begin{aligned}
A_{e} & =\left[\begin{array}{cll}
-0.7959 & 0.06702 & 0.2262 \\
-8.205 & 0.8825 & 0 \\
-10.29 & 0 & 0.9048
\end{array}\right] \\
B_{e} & =\left[\begin{array}{c}
1.701 \\
8.205 \\
10.29
\end{array}\right] \\
C_{e} & =I_{3}, \quad D_{e}=0
\end{aligned}
$$

with performance $J_{e}=0.0358515$.

Permitting nonzero feedthrough $D_{e}$, yields

$$
\begin{aligned}
A_{e} & =\left[\begin{array}{clc}
0.09885 & 0.1061 & -0.0167 \\
-0.6851 & 0.02176 & 0.09015 \\
0.1503 & 0.2652 & 0.8707
\end{array}\right], B_{e}=\left[\begin{array}{c}
-13.34 \\
-10.08 \\
2.237
\end{array}\right] \\
C_{e} & =\left[\begin{array}{clc}
-0.006261 & -0.007282 & 0.0007445 \\
0.5716 & -0.0002166 & 0.007202 \\
0.6822 & -0.006021 & -0.005440
\end{array}\right], \\
D_{e} & =\left[\begin{array}{c}
0.9068 \\
9.298 \\
11.37
\end{array}\right]
\end{aligned}
$$

where the (improved) performance is $J_{e}=0.032401049$.

For the reduced-order results, an algorithm for solving all three equations (31-33) is described briefly. Begin by setting $\tau=I_{n}$ and solving Eqs. (31-33) for the "full-order" values of $Q, \hat{Q}$, and $\hat{P}$. Choose $n_{e}$ eigenprojections of $\hat{Q} \hat{P}$ in diagonalizing coordinates and iterate the modified Lyapunov equations (32) and (33) until convergence of $\tau, \hat{Q}$, and. $\hat{P}$ is obtained. Return to Eq. (31) and solve for $Q$ with $V_{1}+\tau_{\perp} \hat{Q} \tau_{\perp}^{T}$ as the new nonhomogeneous term in the Riccati equation. Repeat the above steps until convergence is reached.

In applying this algorithm to the present example, the eigenprojections were chosen for convenience in accordance with the largest eigenvalues of $\hat{Q} \hat{P}$. The results indicate attainment of the global minimum. For the optimal second-order filter, the gains are given by

$$
\begin{aligned}
& A_{e}=\left[\begin{array}{ll}
0.09898 & -0.1137 \\
0.6632 & -0.02285
\end{array}\right], \quad B_{e}=\left[\begin{array}{c}
-13.33 \\
9.762
\end{array}\right] \\
& C_{e}=\left[\begin{array}{cc}
-0.006259 & 0.007796 \\
0.5716 & -0.0002737 \\
0.6823 & 0.006121
\end{array}\right], \quad D_{e}=\left[\begin{array}{c}
0.9068 \\
9.298 \\
11.37
\end{array}\right]
\end{aligned}
$$


with $J_{e}=0.032401094$, and, for the first-order filter,

$$
\begin{aligned}
A_{e} & =0.1498, \quad B_{e}=-14.82 \\
C_{e} & =\left[\begin{array}{c}
-0.001661 \\
0.5748 \\
0.6897
\end{array}\right], \quad D_{e}=\left[\begin{array}{c}
0.9749 \\
9.489 \\
11.65
\end{array}\right]
\end{aligned}
$$

with $J_{e}=0.03240418$. Convergence to this accuracy was obtained with 7 iterations of Eqs. (31-33) for the second-order filter and 10 iterations for the first-order filter. Note that the performance degrades only slightly with reduced order, and the static gain term gives the first-order filter better performance than the standard (full-order) Kalman filter.

To illustrate Theorem 2.3 for designing reduced-order dynamic compensators, consider a simply supported beam with two colocated sensor/actuator pairs. Assuming the beam has length 2 and that the sensor/actuator pairs are placed at coordinates $a=55 / 172$ and $b=46 / 43$, a continuous-time model of the following form is obtained:

$$
\dot{x}=\hat{A} x+\hat{B} u+\hat{w}_{1} \quad y=\hat{C} x+\hat{w}_{2}
$$

where, retaining the first five modes,

$$
\begin{aligned}
\hat{A}=\text { block-diag }\left(\left[\begin{array}{cc}
0 & 1 \\
-\omega_{1}^{2} & -2 \zeta \omega_{1}
\end{array}\right], \ldots,\left[\begin{array}{cc}
0 & 1 \\
-\omega_{5}^{2} & -2 \zeta \omega_{5}
\end{array}\right]\right) \\
\omega_{i}=i^{2} ; \quad i=1, \ldots, 5, \quad \zeta=0.005 \\
\hat{B}_{(i, 1)}=0.5\left(1+(-1)^{i}\right) \sin (i \pi b / 2), \quad i=1, \ldots, 10 \\
\hat{B}_{(i, 2)}=-0.5\left(1+(-1)^{i}\right) \sin (i \pi a / 2), \quad i=1, \ldots, 10 \\
\hat{C}=\hat{B}^{T}
\end{aligned}
$$

The intensities $\hat{V}_{1}$ and $\hat{V}_{2}$ of $\hat{w}_{1}$ and $\hat{w}_{2}$ are chosen to be

$$
\hat{V}_{1}=0.1 I_{10}, \quad \hat{V}_{2}=0.01 I_{2}
$$

and it is assumed that $\hat{w}_{1}$ and $\hat{w}_{2}$ are uncorrelated. For the continuous-time cost

$$
J=\lim _{t \rightarrow \infty} E\left[x^{T} \hat{R}_{1} x+2 x^{T} \hat{R}_{12} u+u^{T} \hat{R}_{2} u\right]
$$

set

$$
\begin{gathered}
\hat{R}_{1}=\text { block-diag }\left(\left[\begin{array}{cc}
1 & 0 \\
0 & 1 / \omega_{1}
\end{array}\right], \ldots,\left[\begin{array}{cc}
1 & 0 \\
0 & 1 / \omega_{5}
\end{array}\right]\right) \\
\hat{R}_{12}=0, \quad \hat{R}_{2}=I_{2}
\end{gathered}
$$

To convert to the discrete-time problem with discretization interval $h$, let ${ }^{36.49}$

$$
\begin{gathered}
A=e^{\hat{A} h}, \quad B=\int_{0}^{h} e^{\hat{A} t} \hat{B} \mathrm{~d} t, \quad C=\hat{C} \\
V_{1}=\int_{0}^{h} e^{\hat{A} t} \hat{V}_{1} e^{\hat{A}^{T} t} \mathrm{~d} t, \quad V_{2}=\hat{V}_{2} \\
R_{1}=\hat{R}_{1}, \quad R_{12}=0, \quad R_{2}=\hat{R}_{2}
\end{gathered}
$$

The design equations (40-43) for the control problem can be solved using exactly the same techniques as in the previous example for the estimation problem. For the strictly proper case $\left(D_{e}=0\right)$, a series of controllers was designed with $n_{c}=$ $1,2, \ldots, 10$, where the $n_{c}=10$ result is the LQG solution. The gains for the case $n_{c}=4$, for example, are given by

$$
\begin{aligned}
A_{c} & =\left[\begin{array}{lrrr}
0.9317 & 0.1572 & -0.2130 & -0.005038 \\
0.0137 & 0.6879 & 0.2519 & 0.4085 \\
0.3330 & -0.0580 & 0.7713 & -0.2602 \\
0.05980 & -0.3297 & 0.3918 & 0.3005
\end{array}\right] \\
B_{c} & =\left[\begin{array}{cc}
-0.4920 & -0.2166 \\
0.6179 & -0.5959 \\
0.2253 & -0.02572 \\
0.07221 & -0.4863
\end{array}\right] \\
C_{c} & =\left[\begin{array}{cccc}
0.05864 & -0.3094 & -0.01815 & 0.2409 \\
-0.1301 & 0.1463 & -0.1945 & -0.07192
\end{array}\right]
\end{aligned}
$$

Figure 1 summarizes the results for each order, where the rms controller performance is given by

$$
J_{x}^{\frac{1}{2}}=E\left[\lim _{k \rightarrow \infty} x(k)^{T} R_{1} x(k)\right]^{\frac{1}{2}}
$$

These results provide a tradeoff study of performance versus controller order that can be used to assess processor requirements.

\section{Conclusion}

Optimality conditions have been obtained for the problems of least-squares, reduced-order (i.e., fixed-order), discrete-time modeling, estimation, and control. These conditions comprise systems of two, three, and four matrix equations, respectively, coupled by an oblique projection which determines the optimal system gains. When the order of the estimator or controller is equal to the order of the plant, the oblique projection becomes the identity matrix and the estimation and control results specialize to the standard discrete-time Kalman filter and linear-quadratic-Gaussian results. The design results are applied to two illustrative examples. For a third-order stirredtank problem, filters of first and second order are obtained, and, for a simply supported Euler beam example with five flexible modes (i.e., 10 states), a series of reduced-order controllers with $1,2, \ldots, 9$ poles is obtained. The latter results illustrate the tradeoff between control-system performance and controller order.

\section{Acknowledgment}

The authors wish to thank Scott W. Greeley for carrying out the computations in Sec. III.

\section{References}

${ }^{1}$ Hyland, D.C., "Optimality Conditions for Fixed-Order Dynamic Compensation of Flexible Spacecraft with Uncertain Parameters," AIAA Paper 82-0312, Jan. 1982.

${ }^{2}$ Hyland, D.C. and Madiwale, A.N., "Fixed-Order Dynamic Compensation Through Optimal Projection," Proceedings of the Workshop on Applications of Distributed System Theory to the Control of Large Space Structures, edited by G. Rodriguez, Jet Propulsion Laboratory, Pasadena, CA, July 1982, pp. 409-427.

${ }^{3}$ Hyland, D.C. "Mean-Square Optimal Fixed-Otder Compensation - Beyond Spillover Suppression," AIAA Paper 82-1403, Aug. 1982.

${ }^{4}$ Hyland, D.C., "The Optimal Projection Approach to Fixed-Order Compensation: Numerical Methods and Illustrative Results," AIAA Paper 83-0303, Jan. 1983.

${ }^{5}$ Hyland, D.C. and Bernstein, D.S., "Explicit Optimality Conditions for Fixed-Order Dynamic Compensation," Proceedings of the 22nd IEEE Conference on Decision and Control, San Antonio, TX, Dec. 1983, pp. 161-165.

${ }^{6}$ Hyland, D.C., "Comparison of Various Controller-Reduction Methods: Suboptimal Versus Optimal Projection," Proceedings of the AIAA Dynamics Specialists Conference, Palm Springs, CA, May 1984, pp. $381-389$

${ }^{7}$ Hyland, D.C. and Bernstein, D.S., "The Optimal Projection Equations for Fixed-Order Dynamic Compensation," IEEE Transactions on A utomatic Control, Vol. AC-29, 1984, pp. 1034-1037. 
${ }^{8}$ Hyland, D.C. and Bernstein, D.S. “The Optimal Projection Approach to Model Reduction and the Relationship Between the Methods of Wilson and Moore," Proceedings of the 23rd IEEE Conference on Decision and Control, Las Vegas, NV, Dec. 1984, pp. 120-126.

${ }^{9}$ Bernstein, D.S. and Hyland, D.C., "The Optimal Projection Equations for Reduced-Order State Estimation," IEEE Transactions on Automatic Control, Vol. AC-30, 1985, pp. 583-585.

${ }^{10}$ Hyland, D.C. and Bernstein, D.S., "The Optimal Projection Equations for Model Reduction and the Relationships Among the Methods of Wilson, Skelton and Moore," IEEE Transactions on A utomatic Control, to appear.

${ }^{11}$ Bernstein, D.S. and Hyland, D.C., "The Optimal Projection Equations for Finite-Dimensional Fixed-Order Dynamic Compensation of Infinite-Dimensional Systems," SIAM Journal on Control and Optimization, to appear.

${ }^{12}$ Looze, D.P. and Sandell, N. R. Jr., "Gradient Calculations for Linear Quadratic Fixed Control Structure Problems," IEEE Transactions on Automatic Control, Vol. AC-25, 1980, pp. 258-285.

${ }^{13}$ Skelton, R.E., "Cost Decomposition of Linear Systems with Application to Model Reduction," International Journal on Control, Vol. 32, 1980, pp. 1031-1055.

${ }^{14}$ Skelton, R.E. and Yousuff, A., "Component Cost Analysis of Large Scale Systems," International Journal on Control, Vol. 37, 1983, pp. 285-304.

is Yousuff, A. and Skelton, R.E., "Controller Reduction by Component Cost Analysis," IEEE Transactions on Automatic Control, Vol AC-29, 1984, pp. 520-530.

${ }^{16}$ Mitra, D., "Analytical Results on the Use of Reduced Models in the Control of Linear Dynamical Systems," Proceedings of IEE, Vol. 116,1969 , pp. 1439-1444.

${ }^{17}$ Wilson, D.A., "Optimum Solution of Model-Reduction Problem," Proceedings of IEE, Vol. 117, 1970, pp. 1161-1165.

${ }^{18}$ Applevich, J.D., "Approximation of Discrete Linear Systems," International Journal on Control, Vol. 17, 1973, pp. 565-575.

${ }^{19}$ Wilson, D.A., "Model Reduction for Multivariable Systems," International Journal on Control, Vol. 20, 1974, pp. 57-64.

${ }^{20}$ Hickin, J. and Sinha, N.K., "Applications of Projective Reduction Methods to Estimation and Control," Journal on Cybernetics, Vol. 8, 1978, pp. 159-181.

${ }^{21}$ Mishra, R.N. and Wilson, D.A., "A New Algorithm for Optimal Reduction of Multivariable Systems," International Journal on Control, Vol. 31, 1980, pp. 443-466.

${ }^{22}$ Kabamba, P.T., "Model Reduction by Euclidean Methods," Journal of Guidance and Control, Vol. 3, 1980, pp. 555-562.

${ }^{23}$ Moore, B.C." "Principal Component Analysis in Linear Systems: Controllability, Observability, and Model Reduction," IEEE Transactions on Automatic Control, Vol. AC-26, 1981, pp. 17-32.

${ }^{24}$ Eitelberg, E., "Model Reduction by Minimizing the Weighted Equation Error," International Journal on Control, Vol. 34, 1981, pp. 1113-1123.

${ }^{25}$ Sims, C.S., "Reduced-Order Modelling and Filtering," Control and Dynamic Systems, Vol. 18, edited by C.T. Leondes, 1982, pp. 55-103.

${ }^{26}$ Simon, K.W. and Stubberud, A.R., "Reduced Order Kalman Filter," International Journal on Control, Vol. 10, 1969, pp. 501-509.

${ }^{27}$ Sims, C.S. and Melsa, J.L., "Specific Optimal Estimation," IEEE Transactions on Automatic Control, Vol. AC-14, 1969, pp. 183-186.

${ }^{28}$ Asher, R.B., Herring, K.D., and Ryles, J.C., "Bias Variance and Estimation Error in Reduced Order Filters," Automatica, Vol. 12 1976, pp. 589-600.

${ }^{29}$ Galdos, J.I. and Gustafson, D.E., "Information and Distortion in Reduced-Order Filter Design," IEEE Transactions on Information Theory, Vol. IT-23, 1977, pp. 183-194.
${ }^{30}$ Fairman, F.W., "Reduced Order State Estimators for DiscreteTime Stochastic Systems," IEEE Transactions on Automatic Control, Vol. AC-22, 1977, pp. 673-675.

${ }^{31}$ Sims, C.S. and Asher, R.B., "Optimal and Suboptimal Results in Full- and Reduced-Order Linear Filtering," IEEE Transactions on Automatic Control, Vol. AC-23, 1978, pp. 469-472.

${ }^{32}$ Wilson, D.A. and Mishra, R.N., "Design of Low Order Estimators Using Reduced Models," International Journal on Control, Vol. 23, 1979, pp. 447-456.

${ }^{33}$ Dombrovskii, U.V., "Method of Synthesizing Suboptimal Filters of Reduced Order for Digital Linear Dynamic Systems," Automation and Remote Control, Vol. 43, 1982, pp. 1483-1489.

${ }^{34}$ Johnson, T.L. and Athans, M., "On the Design of Optimal Constrained Dynamic Compensators for Linear Constant Systems," IEEE Transactions on Automatic Control, Vol. AC-15, 1970, pp. 658660 .

${ }^{35}$ Levine, W.S., Johnson, T.L., and Athans, M., "Optimal Limited State Variable Feedback Controllers for Linear Systems," IEEE Transactions on Automatic Control, Vol. AC-16, 1971, pp. 785-793.

${ }^{36}$ Kwakernaak, K. and Sivan, R., Linear Optimal Control Systems, Wiley-Interscience, New York, 1972.

${ }^{37}$ Rom, D.B. and Sarachik, P.E., "The Design of Optimal Compensators for Linear Constant Systems with Inaccessible States," IEEE Transactions on Automatic Control, Vol. AC-18, 1973, pp. 509512.

${ }^{38}$ Sidar, M. and Kurtaran, B.Z., "Optimal Low-Order Controllers for Linear Stochastic Systems," International Journal on Control, Vol. 22,1975 , pp. $377-387$

${ }^{39}$ Mendel, J.J., and Feather, J., "On the Design of Optimal TimeInvariant Compensators for Linear Stochastic Time-Invariant Systems," IEEE Transactions on Automatic Control, Vol. AC-20, 1975, pp. 653-657.

${ }^{40}$ Basuthakur, S. and Knapp, C.H., "Optimal Constant Controllers for Stochastic Linear Systems," IEEE Transactions on Automatic Control, Vol. AC-20, 1975, pp. 664-666.

${ }^{41}$ Asher, R.B. and Durrett, J.C., "Linear Discrete Stochastic Control with a Reduced-Order Dynamic Compensator," IEEE Transactions on Automatic Control, Vol. AC-21, 1976, pp. 626-627.

${ }^{42}$ Naeije, W.J. and Bosgra, O.H., "The Design of Dynamic Compensators for Linear Multivariable Systems," 1977 IFAC, Fredricton, Canada, pp. 205-212.

${ }^{43}$ Wenk, C.J. and Knapp, C.H., "Parameter Optimization in Linear Systems with Arbitrarily Constrained Controller Structure," IEEE Transactions on Automatic Control, Vol. AC-25, 1980, pp. 496-500.

${ }^{44}$ O'Reilly, J. "Optimal Low-Order Feedback Controllers for Linear Discrete-Time Systems," Control and Dynamic Systems, Vol. 16, edited by C.T. Leondes, Academic Press, New York, 1980, pp. 335-367.

${ }^{45}$ Grimble, M.J., "Reduced-Order Optimal Controller for DiscreteTime Stochastic Systems," Proceedings of IEE, Vol. 127, 1980, pp. 55-63.

${ }^{46}$ Kabamba, P.T. and Longman, R.W., "An Integrated Approach to Reduced-Order Control Theory," Optimal Control Applications and Methods, Vol. 4, 1983, pp. 405-415.

${ }^{47}$ Kato, T., Perturbation Theory for Linear Operators, 2nd Ed., Springer-Verlag, New York, 1976.

${ }^{48}$ Rao, C.R. and Mitra, S.K., Generalized Inverse of Matrices and Its Applications, John Wiley \& Sons, New York, 1971.

${ }^{49}$ Van Loan, C.F., "Computing Integrals Involving the Matrix Exponential," IEEE Transactions on Automatic Control, Vol. 23, 1978, pp. 395-404. 